PROCEEDINGS OF THE

AMERICAN MATHEMATICAL SOCIETY

Volume 125, Number 4, April 1997, Pages 1177-1185

S 0002-9939(97)03652-6

\title{
CONTINUOUS FUNCTIONS ON COMPACT GROUPS
}

\author{
DAVID P. BLECHER
}

(Communicated by J. Marshall Ash)

\begin{abstract}
We show that every scalar valued continuous function $f(g)$ on a compact group $G$ may be written as $f(g)=\langle\Phi(g) \xi, \eta\rangle$ for all $g \in G$, where $\xi, \eta$ are vectors in a separable Hilbert space $\mathcal{H}$, and $\Phi(g)$ is a strongly continuous unitary valued function on $G$ which is a product of unitary representations and antirepresentations of $G$ on $\mathcal{H}$. This product is countable, but always converges uniformly on $G$. Moreover the supremum norm of $f$ is matched by $\|\xi\|\|\eta\|$. This may be viewed as a 'Fourier product representation' for $f$, and complements a result of Eymard for the Fourier algebra. For 'Fourier polynomials' we show that the Hilbert space may be taken to be finite dimensional, and the product finite, which is more or less obvious except in that we are able to match the correct norm. The main ingredients of the proof are the Peter-Weyl theory, Tannaka's duality theorem, and a method developed with Paulsen using a characterization of operator algebras due to the author, Ruan and Sinclair. We also give the analogues of these formulae for compact quantum groups.
\end{abstract}

Let $G$ be a compact group. Consider the following two important commutative Banach algebras of continuous $\mathbb{C}$ valued functions on $G$. The first is the algebra $C(G)$ of all continuous functions on $G$, with norm $\|f\|_{C(G)}=\max \{|f(g)|: g \in G\}$. The second is the Fourier algebra $A(G)(=B(G)$ in this case) [6]. This may be characterized in several ways. Here are three: as the linear span of the continuous positive definite functions on $G$, as the dual space of the group $\mathrm{C}^{*}$-algebra $\mathrm{C}^{*}(G)$ of $G$, or as the predual of the group von Neumann algebra of $G$. If $G$ is abelian, with (discrete) dual group $\Gamma$, then $A(G)=\ell_{1}(\Gamma)$, where is the Fourier transform. The norm on $A(G)$ in this case coincides with the $\ell_{1}$ norm. Thus the Fourier algebra $A(\mathbb{T})$ of the circle group $\mathbb{T}$ is the set of functions on the circle of form $\sum_{k=-\infty}^{\infty} a_{k} e^{i k \theta}$ where $\|f\|_{A(\mathbb{T})}=\sum_{k=-\infty}^{\infty}\left|a_{k}\right|<\infty$.

In general, we have the following theorem which appears in Eymard [6]:

Theorem 1. A scalar valued function $f$ defined on a compact group $G$ is in $A(G)$ if and only if there exists a strongly continuous unitary representation $\pi$ of $G$ on a Hilbert space $\mathcal{H}$, and vectors $\xi, \eta$ in $\mathcal{H}$, such that $f(g)=\langle\pi(g) \xi, \eta\rangle$. In this case $\xi, \eta$ can be chosen such that $\|f\|_{A(G)}=\|\xi\|\|\eta\|$.

The Hilbert space can be taken to be separable in the above, by a standard argument. The main theorem of this paper is the following complement to the above:

Received by the editors May 25, 1995 and, in revised form, October 26, 1995.

1991 Mathematics Subject Classification. Primary 43A77, 43A25; Secondary 46L05, 43A15.

Supported by a grant from the NSF.

(C)1997 American Mathematical Society 
Theorem 2. A scalar valued function $f$ defined on a compact group $G$ is in $C(G)$ if and only if there exist a sequence of strongly continuous unitary representations and antirepresentations (we can choose these to alternate) $\pi_{n}$ of $G$ on a separable Hilbert space $\mathcal{H}$, and vectors $\xi, \eta$ in $\mathcal{H}$, such that $f(g)=\left\langle\pi_{1}(g) \pi_{2}(g) \cdots \xi, \eta\right\rangle$ for all $g \in G$. In addition, $\|f\|_{C(G)}=\inf \{\|\xi\|\|\eta\|\}$, where the infimum is taken over all such representations of $f$.

Here is what the '...' in the theorem above means: Firstly, the $\pi_{n}$ converge strong-uniformly to the identity operator on $\mathcal{H}$ (that is: for all $\zeta \in \mathcal{H}$, we have $\sup \left\{\left\|\pi_{n}(g) \zeta-\zeta\right\|: g \in G\right\} \rightarrow 0$ as $\left.n \rightarrow \infty\right)$. We shall see that in fact it can be arranged that $\pi_{n}(g)=I d_{\mathcal{H}}$ except on a part of the Hilbert space that becomes insignificant as $n \rightarrow \infty$. Secondly, $f$ is the uniform limit on $G$ of the finite 'truncations' $f_{n}(g)=\left\langle\pi_{1}(g) \pi_{2}(g) \cdots \pi_{n}(g) \xi, \eta\right\rangle$, each of which is a continuous scalar valued function on $G$. Or we can interpret the theorem as saying that $f(g)=\langle\Phi(g) \xi, \eta\rangle$, where $\Phi: G \rightarrow \mathcal{U}(\mathcal{H})$ is the strong-uniform limit of the sequence of (strongly continuous) partial products $\pi_{1}(g) \pi_{2}(g) \cdots \pi_{n}(g)$. Here $\mathcal{U}(\mathcal{H})$ is the group of unitary operators on $\mathcal{H}$. Thus, for each $\zeta \in \mathcal{H}$, we have $\sup \left\{\left\|\pi_{1}(g) \pi_{2}(g) \cdots \pi_{n}(g) \zeta-\Phi(g) \zeta\right\|: g \in G\right\} \rightarrow 0$ as $n \rightarrow \infty$. We are not certain if the presence of the antirepresentations is necessary in the above; in any case for an abelian group there is no such distinction. Thus we have 'stored' all the data of the continuous function as a sequence of representations, and two Hilbert space vectors, in a way that matches the correct norm (within $\epsilon$ ).

We can be a little bit more specific about the form of the $\pi_{n}$ : it will emerge from the proof that each $\pi_{n}$ can be chosen to be a countable direct sum $\bigoplus_{k=1}^{\infty} \pi_{n}^{k}$ of finite dimensional continuous representations (resp. antirepresentations) $\pi_{n}^{k}$ of $G$. Moreover for any $k$, the Euclidean space on which $\pi_{n}^{k}$ acts is the same for all $n$; call this space $\mathcal{H}_{k}$. Then $\mathcal{H}=\bigoplus_{k=1}^{\infty} \mathcal{H}_{k}$. Also, given $k$, there exists an integer $m_{k}$ such that for all $n>m_{k}, \pi_{n}^{k}=I d_{\mathcal{H}_{k}}$. W.l.o.g. $m_{k}$ is increasing. Thus we can write each $\pi_{n}(g)$ as an infinite matrix consisting of two blocks, an upper left hand block which is a direct sum of copies of the trivial representation $\rho(g)=1$, and a lower right hand block. As $n$ increases, the upper left block will take up more and more of the matrix. Let us call a sequence $\left\{\pi_{n}\right\}$ of maps $G \rightarrow \mathcal{U}(\mathcal{H})$ satisfying the conditions of this paragraph a sweeping sequence (for lack of a better name). Such a sequence has the nice property that $\Phi(g)=$ stronguniform $\lim _{n \rightarrow \infty} \pi_{1}(g) \pi_{2}(g) \cdots \pi_{n}(g)$ always exists, and $\Phi$ is strongly continuous. This is because for $n>m_{k}$, we have that $\pi_{1}(g) \cdots \pi_{n}(g)$ restricted to $\mathcal{K}_{k}=\mathcal{H}_{1} \oplus$ $\cdots \oplus \mathcal{H}_{k}$ is independent of $n$, and if $P_{k}^{\perp}$ is the projection of $\mathcal{H}$ onto $\mathcal{K}_{k}^{\perp}$, then $\left\|\pi_{1}(g) \cdots \pi_{n}(g) P_{k}^{\perp} \zeta\right\|=\left\|P_{k}^{\perp} \zeta\right\| \rightarrow 0$ as $n$ (and $\left.k\right) \rightarrow \infty$ independently of $g \in G$. Similarly, the strong-uniform $\lim _{n \rightarrow \infty} \pi_{n}(g)^{*} \pi_{n-1}(g)^{*} \cdots \pi_{1}(g)^{*}$ always exists and equals $\Phi(g)^{*}$. We leave this assertion to the reader. Then for fixed $g$, since $\Phi(g)$ and $\Phi(g)^{*}$ are clearly isometries we see that $\Phi(g)$ is in $\mathcal{U}(\mathcal{H})$. It follows that, if the reader prefers, a continuous function $f$ may be written as $\left\langle\cdots \theta_{2}(g) \theta_{1}(g) \xi, \eta\right\rangle$, where $\left\{\theta_{n}\right\}$ is a sweeping sequence, with an obvious similar interpretation of the '...'. Or it can be arranged so that $f=\left\langle\pi_{1}(g) \pi_{2}(g) \cdots \theta_{2}(g) \theta_{1}(g) \xi, \eta\right\rangle$, which is more symmetrical.

We should also point out here that that $\|f\|_{C(G)} \leq \inf \{\|\xi\|\|\eta\|\}$ is quite obvious (assuming that $f$ has some such representation). The surprizing features are that all continuous functions have such a representation, and the norm equality. Note also that $f(1)=\langle\xi, \eta\rangle$, in both theorems above. It is tempting to think that a fixed 
finite number (say 2) of representations and antirepresentations will be sufficient in Theorem 2, but we can show that this is not the case.

We can deduce from Stone's theorem that:

Corollary 3. $f \in C(\mathbb{T})$ if and only if $f\left(e^{i \theta}\right)=\left\langle e^{i \theta H_{1}} e^{i \theta H_{2}} \cdots \xi, \eta\right\rangle$, for selfadjoint, possibly unbounded, operators $H_{1}, H_{2}, \cdots$ with spectrum contained in $\mathbb{Z}$, and hence which may be diagonalized with integer entries, such that 'more and more' of $H_{n}$ is 0 as $n \rightarrow \infty$. Also $f$ has the same norm formula as above.

The 'more and more' above simply means that as before we can write the Hilbert space $\mathcal{H}=\bigoplus_{k=1}^{\infty} \mathcal{H}_{k}$, and that $H_{n}=0$ on $\bigoplus_{k=1}^{m} \mathcal{H}_{k}$, where $m \rightarrow \infty$ as $n \rightarrow \infty$.

Let us write $M_{N}$ for the $N \times N$ scalar matrices, with the usual operator norm. If $B$ is an algebra of functions on $G$, then $M_{N}(B)$ is the algebra of matrices with entries in $B$, which can obviously be interpreted as an algebra of $M_{N}$-valued functions on $G$. Both $M_{N}(A(G))$ and $M_{N}(C(G))$ have natural norms, the former the natural matrix norms from the 'standard dual' (see [1] for example) of $\mathrm{C}^{*}(G)$, the latter its usual $\mathrm{C}^{*}$-norm $\max \left\{\left\|\left[f_{i j}(g)\right]\right\|_{M_{N}}: g \in G\right\}$. Both theorems above have matrix valued versions. Namely, a function $f: G \rightarrow M_{N}$ is in $M_{N}(A(G))\left(\operatorname{resp} . M_{N}(C(G))\right)$ iff we can write $f(g)=S \pi(g) T$ (resp. $S \Phi(g) T$ where $\Phi$ is as above), where $S: \mathcal{H} \rightarrow$ $\mathbb{C}^{N}, T: \mathbb{C}^{N} \rightarrow \mathcal{H}$ are bounded operators, and again the correct norm is given by $\inf \{\|S\|\|T\|\}$ where the infimum is taken over all such representations of $f$. In the $M_{N}(A(G))$ case the proof follows immediately from the representation theorem for completely bounded maps [8], as is pointed out in [1], and in this case the infimum is achieved (it probably is not in the other case). It is not hard to see how to extend the theorem to maps from $G$ into the compact operators on a Hilbert space, or maps from $G$ into a Hilbert space.

Theorem 2 works well with respect to the algebraic structure of $C(G)$. Namely, it is easy to add, multiply, or take complex conjugates of the 'Fourier product' form, whilst matching the norm. For instance, to multiply $f(g)=\left\langle\pi_{1}(g) \pi_{2}(g) \cdots \xi, \eta\right\rangle$ and $f^{\prime}(g)=\left\langle\theta_{1}(g) \theta_{2}(g) \cdots \zeta, \mu\right\rangle$, simply set $\omega_{k}(g)=\pi_{k}(g) \otimes \theta_{k}(g)$; then we see that $\left(f f^{\prime}\right)(g)=\left\langle\omega_{1}(g) \omega_{2}(g) \cdots(\xi \otimes \zeta),(\eta \otimes \mu)\right\rangle$. Addition of functions corresponds to taking direct sums of the representations, whilst complex conjugation corresponds to conjugating the representations. All three processes can be shown to preserve the 'sweeping' property, and do not disturb the norm (for instance in the product above, $\|\xi \otimes \zeta\|\|\eta \otimes \mu\| \leq\|\xi\|\|\eta\|\|\zeta\|\|\mu\|$ as required).

To prove the main theorem we need to recall some facts from Peter-Weyl theory (see e.g. $[14,7]$ ). The notation below will be used in the rest of the paper without comment. Every compact group $G$ possesses a maximal family $\mathcal{R}$ of mutually inequivalent irreducible finite dimensional continuous unitary representations. Let us write a general element of $\mathcal{R}$ as $u^{\alpha}$ or $\left[u_{i j}^{\alpha}(g)\right]$. The latter for fixed $g$ is a finite matrix of scalars. We have that the trivial representation, which we shall write as $\rho(g)$, is in $\mathcal{R}$, and that if $u^{\alpha}=\left[u_{i j}^{\alpha}(g)\right]$ is in $\mathcal{R}$ then its conjugate representation $\bar{u}^{\alpha}=\left[\overline{u_{i j}^{\alpha}(g)}\right]$ is also in $\mathcal{R}$. Every strongly continuous unitary representation of $G$ is unitarily equivalent to a direct sum of representations in $\mathcal{R}$.

Let $\mathcal{A}_{0}$ be the important subalgebra of $C(G)$ consisting of the Fourier polynomials on $G$. Namely, $\mathcal{A}_{0}$ is the algebra generated by the entries $u_{i j}^{\alpha}(g)$ of these matrix representations. It can be shown that $\mathcal{A}_{0}$ separates points of $G$, and is consequently dense in $C(G)$ by Stone-Weierstrass. Also $\mathcal{A}_{0}$ is simply the linear span of the $u_{i j}^{\alpha}(g)$. It is interesting, although we shall not use this fact, that $\mathcal{A}_{0}$ can be characterized in many ways without reference to representations. For instance, it is the set of 
continuous functions $f$ such that the span of the translates ${ }_{g} f$, for $g \in G$, is finite dimensional.

Set $\Psi$ to be the set of characters of $\mathcal{A}_{0}$, namely the set of homomorphisms $\chi: \mathcal{A}_{0} \rightarrow \mathbb{C}$ such that $\chi(\rho)=1$ and $\overline{\chi(p)}=\chi(\bar{p})$ for all $p \in \mathcal{A}_{0}$. Certainly $G$ is contained in $\Psi$ via 'evaluation at a point in $G$ ': Tannaka's duality theorem [14] states that any $\chi \in \Psi$ is of this form, so that $\Psi=G$.

As an example let $G=\mathbb{T}$; then $\mathcal{R}=\left\{e^{i k \theta}: k \in \mathbb{Z}\right\}$, and $\mathcal{A}_{0}$ is the usual algebra of 'finite' Fourier series.

A final notation: the symbol $A \oplus B$ shall always mean the operator direct sum

$$
\left(\begin{array}{cc}
A & 0 \\
0 & B
\end{array}\right)
$$

except if $A$ and $B$ are Hilbert spaces or vectors in Hilbert space, in which case it means the Hilbert space direct sum.

Theorem 2 will follow from the last assertion of

Theorem 4. A function $f: G \rightarrow \mathbb{C}$ is in $\mathcal{A}_{0}$ if and only if we can write $f(g)=$ $\langle\Phi(g) \xi, \eta\rangle$ for all $g \in G$, where $\xi, \eta$ are vectors in a finite dimensional Hilbert space $\mathcal{H}$, and $\Phi$ is a product of a finite number of continuous unitary representations and antirepresentations of $G$ on $\mathcal{H}$. In addition, $\|f\|_{C(G)}=\inf \{\|\xi\|\|\eta\|\}$, where the infimum is taken over all such representations of $f$.

Again, the matrix valued version of this theorem is valid, and it turns out that we need to prove this version anyway in our proof of Theorem 4.

We can replace the general representations (resp. antirepresentations) $\pi_{n}(g)$ in Theorems 2 and 3 by ones of a more special form, namely those which are a direct sum of the $u^{\alpha}(g)$ (resp. $u^{\alpha}(g)^{-1}$ ) from the Peter-Weyl theory. However to compensate for this gain one is forced to add contractive (or unitary) 'bridging operators', between the $\pi_{n}$. For example, on the circle $\mathbb{T}$ we have that $f \in C(\mathbb{T})$ if and only if $f=\left\langle D_{1}(\theta) T_{1} D_{2}(\theta) T_{2} \cdots \xi, \eta\right\rangle$, where each $D_{k}(\theta)$ is an infinite diagonal matrix with integer powers of $e^{i \theta}$ on the diagonal, and the $T_{k}$ are operators on the separable Hilbert space $\mathcal{H}$ of norm 1 (or $\leq 1$ ) (which may be taken to be unitary). Again, the $D_{k}(\theta), T_{k}$ have the sweeping property. This is made clear below.

Proof of Theorem 4. We shall begin by proving the last remark. We will introduce three 'norms' on the dense commutative subalgebra $\mathcal{A}_{0}$ of $C(G)$, and more generally, defined for $F \in M_{N}\left(\mathcal{A}_{0}\right)$. That they are really norms is not hard, and will emerge from the method of proof (see also [3, 2]). We shall show these norms are the same, which will establish the theorem and the remark before the start of the proof. The first norm will be the usual $C^{*}$-norm $\|\cdot\|_{\infty}$, namely the norm of $F$ viewed as a continuous function on the group. The second 'norm' $\|F\|_{n e w}$ is defined to be $\inf \{\|S\|\|T\|\}$, the infimum taken over all ways to write $F=S \pi_{1}(g) \pi_{2}(g) \cdots \pi_{n}(g) T$, where the $\pi_{k}$ are continuous unitary representations or antirepresentations (chosen to alternate) of $G$ on a finite dimensional Hilbert space $\mathcal{H}$, and where $S$ and $T$ are bounded operators from $\mathcal{H}$ to $\mathbb{C}^{N}$ and from $\mathbb{C}^{N}$ to $\mathcal{H}$ respectively. That every element in $M_{N}\left(\mathcal{A}_{0}\right)$ can be written this way will be demonstrated presently. The third 'norm' $|\cdot|$ is defined to be $\inf \{\|S\|\|T\|\}$, the infimum taken over all ways to write $F=S \pi_{1}(g) A_{1} \pi_{2}(g) A_{2} \cdots \pi_{n}(g) T$, where $\pi_{1}, \pi_{3}, \cdots\left(\right.$ resp. $\left.\pi_{2}, \pi_{4}, \cdots\right)$ are each a finite direct sum of a finite number of the $u^{\alpha}(g)$ (resp. $\left.u^{\alpha}(g)^{-1}\right)$ from the PeterWeyl theory, and the $A_{k}$ and $S, T$ are finite scalar matrices of any size so that the product makes sense, $\left\|A_{k}\right\| \leq 1$. It is quite easy to see that any element of $M_{N}\left(\mathcal{A}_{0}\right)$ 
has such a form. This is because $\mathcal{A}_{0}$ is the span of elements $u_{i j}^{\alpha}(g)=\left\langle u^{\alpha}(g) e_{j}, e_{i}\right\rangle$ which are of the required form; and so it is clearly enough to show that a sum of $S \pi_{1}(g) A_{1} \pi_{2}(g) A_{2} \cdots \pi_{n}(g) T$ and $S^{\prime} \pi_{1}^{\prime}(g) A_{1}^{\prime} \pi_{2}^{\prime}(g) \cdots \pi_{m}^{\prime}(g) T^{\prime}$ is again of this form. By adding copies of the identity matrix we can assume $n=m$, and then we set $\pi_{k}^{\prime \prime}(g)=\pi_{k}(g) \oplus \pi_{k}^{\prime}(g), A_{k}^{\prime \prime}=A_{k} \oplus A_{k}^{\prime}, S^{\prime \prime}=\left[\begin{array}{ll}S & S^{\prime}\end{array}\right]$, and $T^{\prime \prime}=\left[\begin{array}{ll}T & T^{\prime}\end{array}\right]^{t}$. The aforementioned sum equals $S^{\prime \prime} \pi_{1}^{\prime \prime}(g) A_{1}^{\prime \prime} \pi_{2}^{\prime \prime}(g) \cdots \pi_{m}^{\prime \prime}(g) T^{\prime \prime}$, which is of the right form. Note that this also gives the triangle inequality for $|\cdot|$ : we can assume w.l.o.g. that $\|S\|=\|T\|$ and $\left\|S^{\prime}\right\|=\left\|T^{\prime}\right\|$; then $\left\|S^{\prime \prime}\right\|\left\|T^{\prime \prime}\right\|=\left\|S S^{*}+S^{\prime} S^{*}\right\|^{\frac{1}{2}}\left\|T^{*} T+T^{\prime *} T^{\prime}\right\|^{\frac{1}{2}} \leq$ $\|S\|^{2}+\left\|S^{\prime}\right\|^{2}=\|S\|\|T\|+\left\|S^{\prime}\right\|\left\|T^{\prime}\right\|$.

It is easy to see that $\|\cdot\|_{\infty}$ is dominated by $|\cdot|$ by the submultiplicativity of the operator norm. Applying the main theorem of [4] to the algebra $\mathcal{A}_{0}$ with matrix norms $|\cdot|$ as in $[3,2]$, we see that the completion of $\mathcal{A}_{0}$ in $|\cdot|$ is a possibly nonselfadjoint commutative operator algebra with identity $\rho$. Let us call this operator algebra $B$. Pick $\left[u_{i j}^{\alpha}(g)\right] \in \mathcal{R}$, and let us suppose that the size of this matrix is $N$. By definition of $|\cdot|$ on $M_{N}\left(\mathcal{A}_{0}\right)$, it is trivial that in $M_{N}\left(\mathcal{A}_{0}\right)$ we have $\left|\left[u_{i j}^{\alpha}(g)\right]\right| \leq 1$ and $\left|\left[\overline{u_{j i}^{\alpha}(g)}\right]\right| \leq 1$ (the latter since $\overline{u_{i j}^{\alpha}(g)}$ is in $\mathcal{A}_{0}$, and $\left[u_{i j}^{\alpha}(g)\right]^{-1}=\left[\overline{u_{j i}^{\alpha}(g)}\right]$ ). However, in $M_{N}\left(\mathcal{A}_{0}\right)$ we have $\left[u_{i j}^{\alpha}(g)\right]\left[\overline{u_{j i}^{\alpha}(g)}\right]=\left[\overline{u_{j i}^{\alpha}(g)}\right]\left[u_{i j}^{\alpha}(g)\right]=I_{n}$. It is well known that if $v, u$ are contractive operators on Hilbert space, and if $u v=v u=I$, then $v, u$ are unitary with $v=u^{*}=u^{-1}$. Thus in the operator algebra $M_{N}(B)$, we have that $\left[u_{i j}^{\alpha}(g)\right]$ is unitary. Clearly $\left[u_{i j}^{\alpha}(g)\right]^{*}$ lies in $M_{N}(B)$, so that the element $u_{i j}^{\alpha}$, considered as an operator in $B$, has adjoint operator (which is $\overline{u_{i j}^{\alpha}(g)}$ by the relation above) in $B$. Since the $u_{i j}^{\alpha}$ densely generate $B$, we see that $B$ is a self-adjoint operator algebra, that is, a $C^{*}$-algebra. Note that by antilinearity, the operator adjoint $*$ in $B$ restricted to $\mathcal{A}_{0}$ is just complex conjugation of a Fourier polynomial. Since $B$ is commutative and unital, it is isomorphic to $C(\Omega)$ for some compact set $\Omega$. However, $\Omega$ may be interpreted as the characters on $B$. The characters on $B$ restrict to characters on $\mathcal{A}_{0}$, so that $\Omega=G$ by Tannaka duality. (We could also deduce this from Eymard's result [6] that the maximal ideal space of $A(G)$ is $G$.) Hence the $|\cdot|$ norm on $\mathcal{A}_{0}$ equals the spectral radius, which equals $\|\cdot\|_{\infty}$. By the uniqueness of $\mathrm{C}^{*}$-norms on $M_{N}(B)$ if you like, $|\cdot|=\|\cdot\|_{\infty}$ on $M_{N}\left(\mathcal{A}_{0}\right)$ too.

The $(\Leftarrow)$ implication of the theorem follows from the basic Peter-Weyl theory. We will now show that $\|\cdot\|_{\text {new }}$ lies between the other two (equal) norms. Suppose that $F \in M_{N}\left(\mathcal{A}_{0}\right),|F|<1$. Then we can write $F=A_{0} \pi_{1}(g) A_{1} \pi_{2}(g) A_{2} \cdots \pi_{n}(g) A_{n}$, where $\left\|A_{k}\right\|<1$ and $\pi_{k}(g)$ is a direct sum of the representations from the PeterWeyl theorem, or their inverses. We now show, as in [5], that w.l.o.g. we can assume that $A_{k}$ is unitary for all $k$. By induction, suppose that we have shown w.l.o.g. that $A_{1}, \cdots, A_{m-1}$ are unitary. Dilate $A=A_{m}$ to a unitary $A_{m}^{\prime}$ twice the size in the standard fashion:

$$
A_{m}^{\prime}=\left(\begin{array}{cc}
A & \sqrt{1-A A^{*}} \\
-\sqrt{1-A^{*} A} & A^{*}
\end{array}\right)
$$

Then $F=A_{0}^{\prime} \pi_{1}^{\prime}(g) A_{1}^{\prime} \pi_{2}^{\prime}(g) \cdots \pi_{n}^{\prime}(g) A_{n}^{\prime}$, where $A_{0}^{\prime}$ is $A_{0}$ with additional columns of zeroes tacked onto the right, $A_{n}^{\prime}$ is $A_{n}$ with additional rows of zeroes tacked underneath, and each $\pi_{k}^{\prime}$ and the other $A_{k}^{\prime}$ are obtained by direct summing a copy of the identity matrix (onto $\pi_{k}$ and $A_{k}$ ).

Now we absorb the $A_{k}$ into the $\pi_{k}$ 's. Namely, $F=A_{0} \theta_{1}(g) \theta_{2}(g) \cdots \theta_{n}(g) T$ where $T=A_{1} A_{2} \cdots A_{n-1} A_{n}, \theta_{1}(g)=\pi_{1}(g), \theta_{2}(g)=A_{1} \pi_{2}(g) A_{1}^{*}, \theta_{3}(g)=A_{1} A_{2} \pi_{3}(g) A_{2}^{*} A_{1}^{*}$, 
and so on. From this we see that $\|F\|_{\text {new }}<1$. Thus $\|\cdot\|_{\text {new }} \leq|\cdot|$. However, $\|\cdot\|_{\infty} \leq\|\cdot\|_{\text {new }}$ by the submultiplicativity of the operator norm.

Proof of Theorem 2. If $f(g)=\left\langle\pi_{1}(g) \pi_{2}(g) \cdots \xi, \eta\right\rangle$ in the sense described after the statement of Theorem 2 , then $f$ is continuous since the uniform limit of a sequence of continuous functions is continuous. It is also straightforward that $\|f\| \leq\|\xi\|\|\eta\|$.

Let $f \in C(G)$ and $\epsilon>0$ be given. Choose $p_{1}$ in $\mathcal{A}_{0}$ with $\left\|f-p_{1}\right\|<\epsilon$, and $\left\|p_{1}\right\|<\|f\|$. Choose $p_{2} \in \mathcal{A}_{0}$ with $\left\|f-p_{1}-p_{2}\right\|<\frac{\epsilon}{2}$ and $\left\|p_{2}\right\|<\epsilon$. Inductively, choose $p_{n} \in \mathcal{A}_{0}$ with $\left\|f-p_{1}-\cdots-p_{n}\right\|<\frac{\epsilon}{2^{n-1}}$ and $\left\|p_{n}\right\|<\frac{\epsilon}{2^{n-2}}$. Then $f=\sum_{k=1}^{\infty} p_{k}$ uniformly. Let us write $p_{k}=\left\langle\pi_{1}^{k}(g) \pi_{2}^{k}(g) \cdots \pi_{m_{k}}^{k}(g) \xi_{k}, \eta_{k}\right\rangle$, as in Theorem 4, where $\left\|\xi_{k}\right\|^{2}=\left\|\eta_{k}\right\|^{2}<\frac{\epsilon}{2^{n-2}}$. By adding an infinite number of copies of the identity after $\pi_{m_{k}}^{k}$ we can assume $m_{k}=\infty$. Let $\pi_{n}=\pi_{n}^{1} \oplus \pi_{n}^{2} \oplus \cdots$ for each $n$, and let $\xi=\xi_{1} \oplus \xi_{2} \oplus \cdots, \eta=\eta_{1} \oplus \eta_{2} \oplus \cdots$. Then $\|\xi\|\|\eta\|<\sqrt{\|f\|^{2}+2 \epsilon}$. It is easy to check that $f(g)=\left\langle\pi_{1}(g) \pi_{2}(g) \cdots \xi, \eta\right\rangle$ in the sense described after the statement of Theorem 2.

The proof of the $M_{n}(C(G))$ case is similar.

Remark 1. Suppose we wish to extend a continuous function $f: G \rightarrow M_{n}$ to a compact group $K$ containing $G$ as a closed subgroup. For notational simplicity let $n=1$. From the method of proof of Theorem 2, it is sufficient to extend a function in $\mathcal{A}_{0}$, namely one of the form $\left\langle\pi_{1}(g) \pi_{2}(g) \cdots \pi_{n}(g) \xi, \eta\right\rangle$ described in Theorem 4 , to a function of close norm. However each representation (and antirepresentation) can be extended to $K$ by Mackey's 'induced representations' theory [7]. Unfortunately they may now act on different Hilbert spaces, but since the original finite dimensional Hilbert space $\mathcal{H}$ is a subspace of each new Hilbert space, we may simply insert a projection onto $\mathcal{H}$ between the $\pi_{k}$ 's. By the 'stages theorem' in induced representation theory [7], this extension process is smooth in the sense that if $K$ is contained in a third group $H$, then if we first extend to $K$ and then to $H$ we obtain the same result as if we extend immediately to $H$. This seems to have some advantage over a simple Tietze extension.

Remark 2. We omit the details, but by the principle mentioned earlier of 'sweeping sequence' implying automatic strong-uniform convergence, a continuous function $f$ on $G$ extends to a continuous function on the compact group $\prod_{k=1}^{\infty} G$ with the Tychonoff topology, by defining $\tilde{f}\left(g_{1}, g_{2}, \cdots\right)=\left\langle\pi_{1}\left(g_{1}\right) \pi_{2}\left(g_{2}\right) \cdots \xi, \eta\right\rangle$.

Remark 3. The reader with some familiarity with completely bounded multilinear maps will notice a similarity between our 'product' form of $f$, and the ChristensenSinclair-Paulsen-Smith factorization form (see for instance [5]) for completely bounded multilinear maps. Indeed, suppose $f \in \mathcal{A}_{0}$. Then

$$
f(g)=\left\langle\pi_{1}(g) \pi_{2}(g) \cdots \pi_{n}(g) \xi, \eta\right\rangle
$$

as in Theorem 4. Suppose $G$ is abelian, for simplicity. Each $\pi_{k}(g)$ extends to a *representation $\pi_{k}^{\prime}$ of $C^{*}(G)$, and so $\tilde{f}\left(g_{1}, \cdots, g_{n}\right)$ (defined as in Remark 2) gives rise to a bounded linear functional $F\left(h_{1} \otimes \cdots \otimes h_{n}\right)=\left\langle\pi_{1}^{\prime}\left(h_{1}\right) \pi_{2}^{\prime}\left(h_{2}\right) \cdots, \pi_{n}^{\prime}\left(h_{n}\right) \xi, \eta\right\rangle$ on the Haagerup tensor product (see [5] for example) $C=C^{*}(G) \otimes_{h} C^{*}(G) \otimes_{h} \cdots \otimes_{h}$ $C^{*}(G)$ of $n$ copies of $C^{*}(G)$. This functional is already written in its ChristensenSinclair factorization form. Also, $F$ extends to the free product $\mathrm{C}^{*}$-algebra $D=$ $C^{*}(G) * C^{*}(G) * \cdots * C^{*}(G)$ of $n$ copies of $C^{*}(G)$. Moreover, the norms of these functionals are within $\epsilon$ of the norm of $f$. Conversely, a functional on $C$ or $D$ 
will (by reversing the process above) give a continuous function on $G$. Such 'finite product' functions on groups have been studied by R. Blei, B. Schreiber, G. Zhao and perhaps others. See $[15,16,17]$ for example.

Remark 4. If $G$ is a locally compact abelian group, then it follows by passing to the Bohr compactification that every continuous almost periodic bounded scalar valued function on $G$ has a representation as in Theorem 2. The converse is not true, though. We are not sure if every continuous bounded scalar valued function on $G$ has such a representation.

We now indicate how to extend the results to compact quantum groups $(\mathcal{A}, \Phi)$ in the sense of Woronowicz $[11,13]$. This is a unital $\mathrm{C}^{*}$-algebra $\mathcal{A}$ with a ${ }_{-}$ homomorphism $\Phi: \mathcal{A} \rightarrow \mathcal{A} \otimes_{\min } \mathcal{A}$ which is coassociative, such that both the span of terms of the form $(a \otimes 1) \Phi(b)$, and terms of the form $(1 \otimes a) \Phi(b)$, are dense in $\mathcal{A} \otimes_{\min } \mathcal{A}$. Coassociativity means that $\left(\Phi \otimes I d_{A}\right) \circ \Phi=\left(I d_{A} \otimes \Phi\right) \circ \Phi$. In [13] it is assumed that $\mathcal{A}$ is separable, or at least has a faithful state, but Van Daele showed that this condition may be removed [10]. Examples of compact quantum groups include $C(G)$ for a compact group $G$, and the full and reduced $\mathrm{C}^{*}$-algebras, $\mathrm{C}^{*}(\Gamma)$ and $\mathrm{C}_{r}^{*}(\Gamma)$, of a discrete group $\Gamma$. An $n$-dimensional unitary representation of a compact quantum group is a unitary matrix $\left[u_{i j}\right]$ in $M_{n}(\mathcal{A})$ such that $\Phi\left(u_{i j}\right)=\sum_{k=1}^{n} u_{i k} \otimes u_{k j}$ for all $i, j$. Let $\mathcal{R}_{n}$ be the set of all $n$-dimensional unitary representations, and $\mathcal{R}=\bigcup_{n=1}^{\infty} \mathcal{R}_{n}$. Woronowicz shows that the Peter-Weyl theory goes through. In particular, the entries of the matrices in $\mathcal{R}$ span a dense *subalgebra $\mathcal{A}_{0}$ of $\mathcal{A}$. We may then define a norm on $\mathcal{A}_{0}$ by $|u|=\inf \{\|\underline{\lambda}\|\|\underline{\mu}\|\}$, where the infimum is taken over all $m, n \in \mathbb{N}$, and ways to write $u=\underline{\lambda}^{t} v_{1} v_{2}^{*} v_{3} v_{4}^{*} \cdots v_{n} \underline{\mu}$ where $\underline{\lambda}, \mu \in \mathbb{C}^{m}$ and $v_{k} \in \mathcal{R}_{m}$ for all $k$. As above in the compact group case, it follows that the completion of $\mathcal{A}_{0}$ in the norm $|\cdot|$ is a $\mathrm{C}^{*}$-algebra $\mathcal{B}$. Note that in the proof of Theorem 4, we did not need to use the inequivalent unitary representations $u^{\alpha}$ from the Peter-Weyl theory, one can use any finite dimensional representations. The norm $|\cdot|$ dominates the original norm clearly, so that $\mathcal{A}$ is a quotient $\mathrm{C}^{*}$-algebra of $\mathcal{B}$. The restriction of $\Phi$ to $\mathcal{A}_{0}$ (which Woronowicz shows has range exactly equal to the algebraic tensor product $\mathcal{A}_{0} \otimes \mathcal{A}_{0}$ ) extends to a map $\mathcal{B} \rightarrow \mathcal{B} \otimes_{\min } \mathcal{B}$, making $(\mathcal{B}, \Phi)$ a compact quantum group. It is easy to see that it is in fact the maximal enveloping compact quantum group with dense ${ }^{*}$-subalgebra $\mathcal{A}_{0}$. For example, if $\mathcal{A}=C_{r}^{*}(\Gamma)$, the reduced $\mathrm{C}^{*}$-algebra of a discrete group, then $\mathcal{B}=C^{*}(\Gamma)$, the full group $\mathrm{C}^{*}$-algebra. (We remark that in this specific case the norm formula on $\mathcal{A}_{0}$ appears in [3].) Thus if we are in an amenable situation (see [9] and the references contained therein), then $\mathcal{B}=\mathcal{A}$ and the norm $|\cdot|$ equals the original norm on $\mathcal{A}_{0}$. We can describe elements of $\mathcal{B}$ as in Theorem 2 , using the same proof. However, in fact we have the formula on $\mathcal{A}$ : that is, $u \in \mathcal{A}$ if and only if $u$ is the limit of a norm convergent product $u=\underline{\lambda}^{t} v_{1} v_{2}^{*} v_{3} v_{4}^{*} \cdots \mu$. Here $\underline{\lambda}, \mu \in \ell_{2}$, and $v_{n}=\bigoplus_{k} v_{n}^{k}$, where $v_{n}^{k} \in \mathcal{R}$, and for fixed $k, v_{n}^{k}$ is the same size matrix for all $n$. We need the 'sweeping' condition guaranteeing convergence in norm as before: for fixed $n, v_{n}^{k}$ is the identity matrix for $k=1, \cdots, m$, where $m$ increases to $\infty$ as $n$ increases. Moreover $\|u\|=\inf \{\|\underline{\lambda}\|\|\mu\| \|\}$, where the infimum is taken over all such products which converge to $u$.

Certainly, the proof of Theorem 2 gives the result as stated for $\mathcal{B}$. However, if $u \in \mathcal{A},\|u\|<1$, then $u$ is the image under the quotient *-homomorphism of an element $U$ in $\mathcal{B},|U|<1$. Then $U$ has such an infinite product form, with $\|\underline{\lambda}\|\|\mu\|<1$. Applying the quotient ${ }^{*}$-homomorphism to the infinite product form 
gives an identical product form which now converges in $\mathcal{A}$ to $u$. The norm formula is now plain.

If one considers the case where $\mathcal{A}=C_{r}^{*}\left(F_{2}\right)$ for instance, it is curious that we seem to get the identical norm formula for elements of $C_{r}^{*}\left(F_{2}\right)$ and of $C^{*}\left(F_{2}\right)$, and of course all $\mathrm{C}^{*}$-algebras in between. But the point is that the infimum giving the $C^{*}\left(F_{2}\right)$ will be over a smaller set; or in other words, a product form which converges to 0 in $C_{r}^{*}\left(F_{2}\right)$ will converge, but not necessarily to zero, in $C^{*}\left(F_{2}\right)$.

The technique of this paper had birth in joint work with Vern Paulsen, and is obviously quite general. We refer to [3], [2] for a survey of related results. Note that in Example 23 of [2] the main results of this paper are summarized without proof: for a compact group $G$ and with $\Lambda$ and $\mathcal{R}$ as described in Example 23, $O A(\Lambda, \mathcal{R})=C(G)$, whereas $O S(\Lambda, \mathcal{R})=A(G)$ in the notation of that paper. It should be noted that some of the 'quantum variable' examples described in section 3 of [2] are 'maximal enveloping' $\mathrm{C}^{*}-$ algebras. One may then play the trick of the last paragraph to obtain factorizations and norm formulae for associated quotient $\mathrm{C}^{*}$-algebras, for example for the minimal $\mathrm{C}^{*}$-algebra tensor product, or for the reduced free product $\mathrm{C}^{*}$-algebra, of two $\mathrm{C}^{*}$-algebras. The generality of this type of result suggests that they are not as useful as the 'finite product' versions which

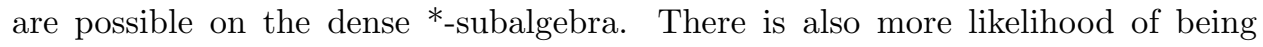
actually able to get one's hands on finite products.

We thank Professor S. Woronowicz for a useful comment, and Jon Kraus for several conversations.

\section{REFERENCES}

1. D. P. Blecher, The standard dual of an operator space, Pacific J. Math. 153 (1992), 15-30. MR 93d:47083

2. __ Factorizations in universal operator spaces and algebras, To appear, Rocky Mountain Math. J.).

3. D. Blecher and V. Paulsen, Explicit construction of universal operator algebras and an application to polynomial factorization, Proc. Amer. Math. Soc. 112 (1991), 839-850. MR 91j:46093

4. D. Blecher, Z-j. Ruan, and A. Sinclair, A characterization of operator algebras, J. Functional Anal. 89 (1990), 188-201. MR 91b:47098

5. E. Christensen, E. Effros, and A. Sinclair, Completely bounded multilinear maps and $C^{*}-$ algebraic cohomology, Inv. Math. 90 (1987), 279-296. MR 89k:46084

6. P. Eymard, L'algebre de Fourier d'un groupe localement compact, Bull. Soc. Math. France 92 (1964), 181-236. MR 37:4208

7. J. M. G. Fell and R. S. Doran, Representations of *-algebras, locally compact groups, and Banach *-algebraic bundles, Vol. 126 in Pure and Appl. Math. Series, Academic Press, 1988. MR 90c: 46002

8. V. Paulsen, Completely bounded maps and dilations, Pitman Research Notes in Math., Longman, London, 1986. MR 88h:46111

9. Z-j. Ruan, Amenability of Hopf von Neumann algebras and Kac algebras, J. Functional Anal. 139 (1996), 466-499.

10. A. Van Daele, The Haar measure on a compact quantum group, Proc. Amer. Math. Soc. 123 (1995), 3125-3128. MR 95m:46097

11. S. Wang, Ph. D Thesis, Berkeley (1993).

12. S. L. Woronowicz, Compact matrix pseudogroups, Comm. Math. Phys. 111 (1987), 613-665. MR 88m:46079

13. Compact quantum groups, Preprint (1993).

14. K. Yosida, Functional Analysis, 3rd Edition, Springer-Verlag, 1971. MR 39:741

15. R. C. Blei, Projectively bounded Frechet measures, Trans. A.M.S. (to appear). 
16. G. Zhao and B. M. Schreiber, Algebras of multilinear forms on groups, Contemp. Math. 189 (1995), 497-511.

17. G. Zhao, Completely bounded multilinear forms on groups, preprint (1995).

Department of Mathematics, University of Houston, Houston, Texas 77204-3476

E-mail address: dblecher@math.uh.edu 